\title{
Improved solutions for dangerous liquid containment
}

\author{
G. Janszen, A. M. Grande, P. Bettini \& L. Di Landro \\ Department of Aerospace Science and Technologies, \\ Politecnico di Milano, Italy
}

\begin{abstract}
The availability of new materials with uncommon characteristics and functionalities gives the opportunity to develop and possibly integrate new conceptual solutions for the improvement of safety in the design and manufacture of containers for critical substances. Numerous examples can be devised where a proper containment of liquid, even after tank damage, is essential with regard to safety issues. Bullet penetration or debris impact of fuel tanks are two relevant examples where specifically designed configuration and materials may significantly improve safety margins. In such situations, deflagration can be activated by sudden variation of internal pressure and temperature or liquid spilling due to wall container perforation.

Cellular filler material in the container can remarkably dispel fire heat and limit pressure wave peaks generated by a bullet explosion; moreover, the overall response to impact loads in case of a crash is remarkably improved. Different fillers with particular configurations, ranging from porous structure to expanded aluminium foil, have been tested as tank explosion suppression media with remarkably positive results.

In traditional multilayer wall fuel tanks, self-sealing capability can be obtained as a result of swelling or chemical reaction of rubber/foam layer when it comes in contact with the spilling internal liquid. A usual drawback of these materials is the limited resistance to aging which reduces their effectiveness with time. Moreover, the sealing material does not give structural contribution furthermore consistently increasing the weight of the component. Polymeric materials with intrinsic self-healing capability may become a valid alternative to traditional configurations. In this context, ethylene-methacrylic acid based ionomers can be adopted as self-healing layers. The coupling of such self-
\end{abstract}


healing polymer with a rigid cellular filler can have multiple benefits such as high impact resistance, repeatable and environmentally stable self-healing capability, lightness, mechanical stiffness and strength.

Keywords: fuel tanks, fillers, self-healing, impact.

\section{Introduction}

One of the causes of fatalities in the case of an aircraft accident is the development of fire in a hard landing or even in a crash event. Fuel tank explosion, which results from ignition of vapours mainly by electric discharges, is the major responsible of this event. The explosions starts primarily from the leakage of the fuel due to the rupture of the fuel tank walls. This means that a possible solution must deal with two issues to be focused on: the leakage of the fuel and the suppression of the explosion. Over the years many concepts, for the prevention or suppression of explosions, have been explored. Among these, foam and metallic fillers are the most used in military aircrafts and helicopters. One of the authors has deeply investigated the behaviour of a particular expanded aluminium foil in the case of a crash event. Several deceleration and drop tests have been carried out. During these tests, acceleration and pressure have been measured and numerical models have been developed to simulate interaction between the fluid and the filler [1,2]. Figures 1 and 2 show the filler and the deceleration tests with and without the filler.

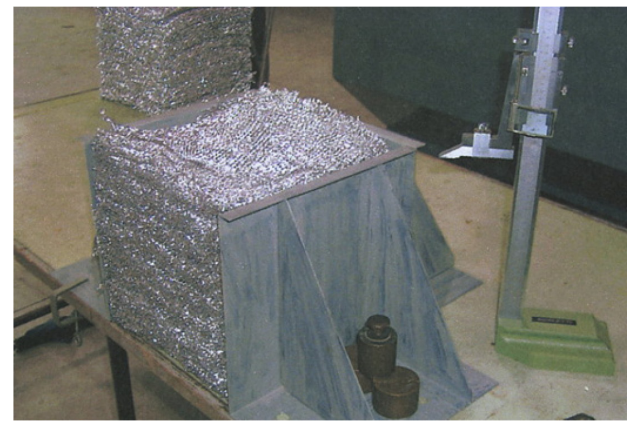

Figure 1: The expanded aluminium foil.

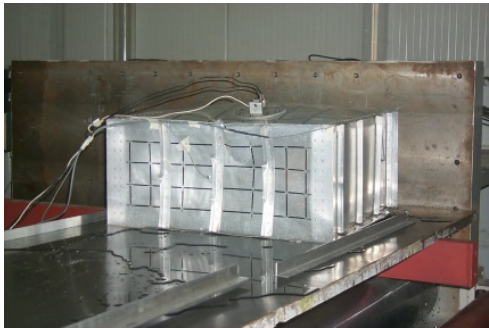

(a)

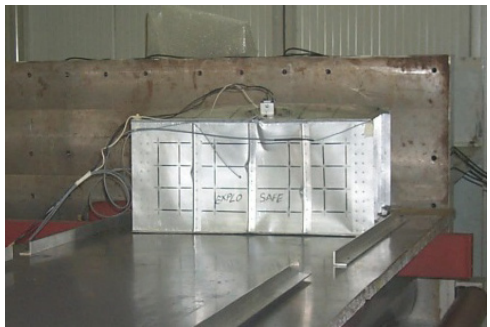

(b)

Figure 2: Deceleration tests with impact, without filler (a) and with filler (b). 
Results of these tests showed an overall good behaviour during impact in terms of wall failure and pressure drop when the filler was present. Unfortunately some leakage was still present. Also in case of bullet perforation, although the cellular filler may significantly reduce the extent of spilling, some potentially dangerous fuel leakage should be expected. This issue brought the authors to evaluate a new fuel tank concept in which both a cellular filler and tank walls made of rigid self-healing polymer or multilayer composite can be employed.

The development of self-healing materials has found important scientific and technological implications, particularly in relation to cost-effective approaches toward damage management of structures. Thermoplastic materials, such as ionomers based on ethylene-co-methacrylic acid copolymers, partially neutralized with sodium or zinc, have shown self-healing behaviour after ballistic impacts [3-9]. Blends of ionomer with different polymers, yet with selfmending ability, were also investigated [10-14]. However, certain conditions in terms of proper temperature range, bullet speed and shape are necessary for the autonomic healing to occur.

Aramid fabrics are widely used for flexible ballistic protections thanks to their tenacity and impact energy absorption capacity, while aramid reinforced composites and foam cored sandwich structures find extensive employment in rigid armour. Carbon foams present particular mechanical and thermal properties, which make them interesting in a number of structural applications $[15,16]$. They show remarkable ballistic performances for relatively small fragments impacts; researchers found that low density carbon foams were able to stop and in some cases hold a $5 \mathrm{~mm}$ diameter stainless steel sphere fired at a speed up to $240 \mathrm{~m} / \mathrm{s}$ by a compressed air gun $[17,18]$. The coupling of aramid fabric or carbon foams with polymers able to restore, at least partially, the continuity of the material may significantly extend the performances and the application areas of such systems.

The self-healing ability of plain ethylene-methacrylic acid copolymer, partially neutralized with sodium ions (EMAANa), and multilayer composites based on the same ionomer have been studied by ballistic puncture tests; different conditions were assessed by varying sample thickness, bullet impact velocity (from $180 \mathrm{~m} / \mathrm{s}$ up to $4000 \mathrm{~m} / \mathrm{s}$ ), bullet shape and diameter [19].

At tested impact speed, EMAANa presented self-repair ability up to a specific sample thickness/projectile diameter ratio even at highest impact speeds. On the other hand, ballistic tests of multilayer systems showed that self-healing behaviour of ionomeric layers can be maintained also in composites, within defined impact speed limits. The analysis of healing efficiency was evaluated by leakage tests and microscopy observations. By applying a pressure gradient trough punctured panels, hole closure and tightness were tested by following vacuum decay and by checking for possible flow of a fluid droplet placed in correspondence of damage. A morphology analysis of the impact zones was made observing all samples by optical stereomicroscope and scanning electron microscope both in the bullet entrance and exit sides. 


\section{Experimental}

\subsection{Materials and samples production}

Ethylene-co-methacrylic acid based copolymers and ionomers are available on the market with different trade names (e.g. Nucrel ${ }^{\circledR}$ and Surlyn ${ }^{\circledR}$ ). In this research an ionomer neutralized with sodium was analysed.

The ethylene-methacrylic acid ionomers, grade Surlyn ${ }^{\circledR} 8940$, was provided by DuPont TM Italy. The polymer is characterized by a content of $5.4 \mathrm{~mol} \%$ acid groups, $30 \%$ of which neutralized with sodium. The density is $0.95 \mathrm{~g} / \mathrm{cm}^{3}$.

After drying received pellets in vacuum at $60^{\circ} \mathrm{C}$ for 5 hours, square samples $(120 \times 120 \mathrm{~mm})$ of $1 \mathrm{~mm}$ and $2 \mathrm{~mm}$ thickness were produced by compression moulding setting the mould temperature at $180^{\circ} \mathrm{C}$. Prior to testing, flat specimens were stored in a controlled environmental chamber at $23^{\circ} \mathrm{C}$ for 30 days in order to achieve non-variable and stable mechanical properties due to aging.

Two different materials were used as reinforcement or core, in particular carbon foam FPA-35, supplied by GrafTech International, with bulk density of $0.56 \mathrm{~g} / \mathrm{cm}^{3}$ and aramid fabric, STYLE 281, provided by Seal SpA.

Ionomeric plates, $120 \times 120 \mathrm{~mm}$, were produced by compression moulding technique using a hot platens hydraulic press. Pelletized polymer was placed in the mould heated at $180^{\circ} \mathrm{C}$ and then pressed in order to obtain plates of different thickness.

Multilayer composites were produced using the same technique; in the specific, previously produced $1 \mathrm{~mm}$ and $2 \mathrm{~mm}$ thickness polymeric plates, were used as external sheets in the stacking sequence of the aramid based hybrid composites and carbon foam core sandwich panels, respectively (Figure 3). All layer were positioned within the mould and then lightly pressed for 10 minutes at $120^{\circ} \mathrm{C}$, in order to allow the adhesion between the different layers. In case of aramid reinforcement partial impregnation of the fabric was obtained.

After productions, all samples were stored in temperature and humidity controlled chamber prior to testing.

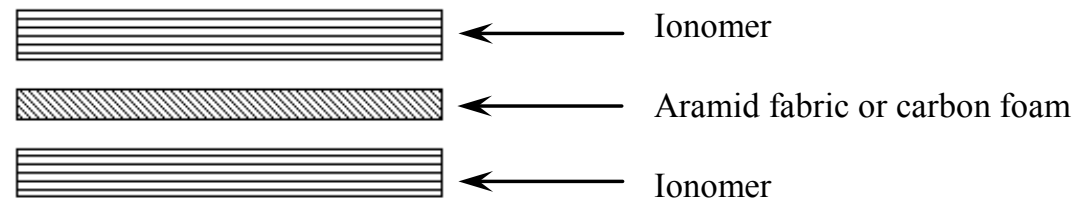

Figure 3: General representation of the different multilayer composites.

\subsection{Ballistic tests and healing evaluation}

Ballistic puncture tests on ionomer plates and multilayer composites were performed at Fiocchi Munizioni Ballistic Laboratory by shooting 4.65x19.2 mm bullets through 120x120 mm square samples. The speed of bullets, measured using a laser beam, ranged between 700 and $730 \mathrm{~m} / \mathrm{s}$. Hypervelocity tests of 
ionomer plates were carried out up $4000 \mathrm{~m} / \mathrm{s}$ with a two-stage Light-Gas Gun; aluminium spheres with $1.5 \mathrm{~mm}$ diameter were used as bullets [19, 20].

Low-velocity $(180 \mathrm{~m} / \mathrm{s})$ and mid-velocity $(400 \mathrm{~m} / \mathrm{s})$ impact tests were performed by shooting steel balls of different sizes using an air gun and a shotgun, respectively [19].

All specimens were observed by optical and scanning electron microscope (SEM) both in the bullet entrance and exit sides to have evidence of hole closures. SEM analyses were also performed to evaluate the morphology of the damaged surfaces of the specimens.

To check for the ability of healing, leakage tests were carried out; a pressure difference of 0.9 bars was initially applied by a vacuum pump in a closed chamber, sealed with the tested polymer plate. Air tightness through the hole was tested following vacuum decay and by checking for possible flow of a fluid droplet placed at the damage zone with the applied pressure difference. When the hole was healed no appreciable vacuum decay was detected within the specified time range but for non-healed samples vacuum decay was observed within a few seconds.

\section{Results and discussion}

Ballistic impact tests showed that in all cases, the bullet energy employed was sufficient to perforate ionomer or multilayer plates. In the latter materials, however, the presence of a reinforcing fabric or foam efficiently adherent to ionomer in some cases reduced the self-healing efficiency. The healing mechanism of ionomers is well discussed in the literature [3-9]. It is the result of a "welding" effect, where the energy dissipation due to material plastic deformation and projectile friction, leads to material melting; the following viscoelastic recovery and material solidification are able to, at least partially, close and seal the damage. An insufficient projectile impact energy or the presence of deformation constraints may impair the healing ability of the material.

Morphological analysis of multilayer panels after ballistic tests showed a different conformation of the impacted sites. It can be noted that in aramid reinforced plate (Figure 4(a)), delaminations in the impact area (white halos) are evidenced. The reduced constraint exerted by the fabric in such areas, allows for ionomeric polymer viscoelastic recovery and efficient healing. A complete hole closure with evidence of polymer melting and full welding can be observed (Figure 5(a)).

Ballistic experiments carried out on carbon foam/EMAANa sandwiches revealed a different behaviour in the self-repairing phenomenon of the ionomeric layers (Figure 4(b)). Hole closure with molten material appeared only in the first EMAANa skin hit by the bullet (inlet layer). When the bullet passed through carbon foam, the outlet ionomeric layer did not exhibited self-healing, as revealed by leakage tests. While the projectile energy is certainly sufficient for the effective healing of the inlet layer, it is conceivable that the passage through the first polymer skin and carbon core, reduces its energy so that no re-welding 
and healing of the outer ionomer layer is possible. A further possible origin of reduced healing capacity of the outer EMAANa layer could be attributed to the carbon microparticles generated during the impact of the projectile with the foam: a cloud of such particles is deposited on the damaged area and may lock the repair process of the ionomer, by preventing the full sealing of the hole.

The results suggest that multilayer configurations for fuel containment can result efficient, provided limited coupling between self-healing and structural layers is reached. On the basis of these considerations, experimental evaluations of the healing ability in sandwich panels made of external ionomer skin, inner composite laminate and with honeycomb core have already given promising results. A next research activity considering coupled ionomer skin/cellular filler and composite wall/cellular filler as fuel containment materials is programmed.

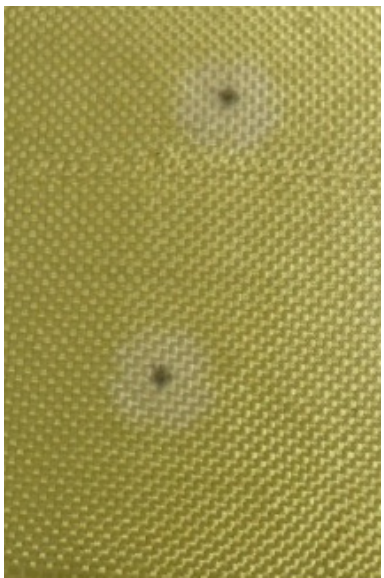

(a)

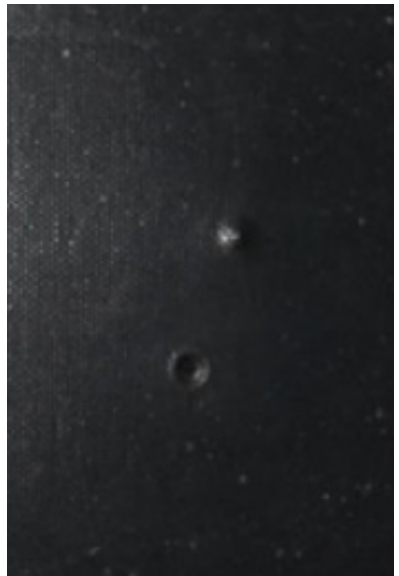

(b)

Figure 4: Aramid fabric/EMAANa (a) and carbon foam/EMAANa (b) multilayer samples tested.

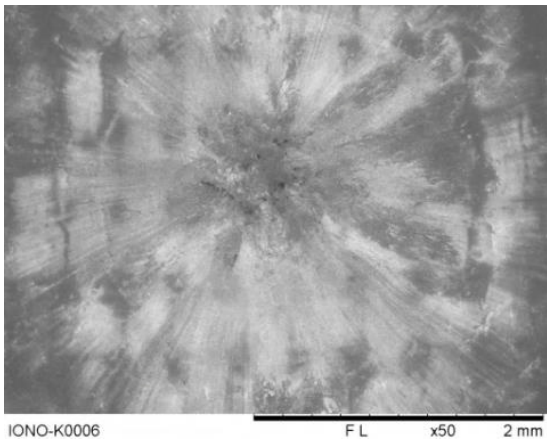

(a)

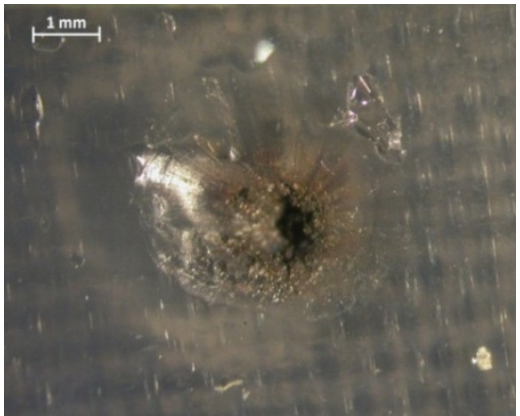

(b)

Figure 5: $\quad$ Bullet Inlet side (a) on the aramid fabric/EMAANa (b) and carbon foam/EMAANa multilayer samples. 


\section{Conclusions}

In this work the self-healing behaviour of different ionomeric systems was explored. Ballistic impact tests on different multilayer composites showed that self-healing behaviour and hole closure was well maintained in all samples; these results encourage the study of ionomeric systems and the development of new complex structures yet able to maintain efficient self-healing ability.

The next step will be the creation of a numerical model in which the coupling of the self-healing material with the expanded aluminium foil filler will show the interaction of both systems in case of a high energy event. The model will be validated by experimental ballistic and drop tests. The self-healing capacity of such configurations will be experimentally checked.

\section{Acknowledgements}

The authors wish to thank Fiocchi Munizioni Ballistic Laboratory, Lecco, Italy, for their support in carrying out the ballistic tests.

The financial support of project SMAT - Regione Lombardia is gratefully acknowledged.

\section{References}

[1] Caprile C., Janszen G. and Morandini M. Study of the effects of a particular fuel tank filler in crash environment in Proceedings of 16th AIDAA National Conference, Palermo, Italy (2001).

[2] Janszen G. and Pernechele A. Experimental analysis and numerical simulation of a fuel tank filler in crash environment in Proceedings of 3rd International Conference on Fluid Structure Interaction, La Coruña, Spain (2005).

[3] Kalista S. J., Ward T. C. and Oyetunji Z. Self-healing of poly(ethylene-comethacrylic acid) copolymers following projectile puncture. Mechanics of Advanced Materials and Structures, 14, pp. 391-397 (2007).

[4] Kalista S. J. and Ward T. C. Thermal characteristics of the self-healing response in poly(ethylene-co-methacrylic acid) copolymers. Journal of the Royal Society Interface, 4, pp. 405-411 (2007).

[5] Varley R. J. and van der Zwaag S. Development of a quasi-static test method to investigate the origin of self-healing in ionomers under ballistic conditions. Polymer Testing. 27, pp. 11-19 (2008).

[6] Varley R. J. and van der Zwaag S. Towards an understanding of thermally activated self-healing of an ionomer system during ballistic penetration. Acta Materialia, 56, pp. 5737-5750 (2008).

[7] Varley R. J. and van, der Zwaag S. Autonomous damage initiated healing in a thermo-responsive ionomer. Polymer International, 59, pp. 1031-1038 (2010).

[8] A. Francesconi, C. Giacomuzzo, A. M. Grande, T. Mudric, M. Zaccariotto, E. Etemadi, L. Di Landro, U. Galvanetto, "Comparison of Self-healing 
Ionomer to Aluminium-alloy Bumpers for Protecting Spacecraft Equipment from Space Debris Impacts”, in Advances in Space Research, 51, 930-940 (2013).

[9] A.M. Grande, L. Castelnovo, L. Di Landro, C. Giacomuzzo, A. Francesconi, Md. A. Rahman, "Rate dependent self-healing behavior of an ethylene-co-methacrylic acid ionomer under high energy impact conditions", in Journal of Applied Polymer Science, J. APPL. POLYM. SCI. 2013, DOI: 10.1002/APP. In press.

[10] Rahman M. A., Penco M., Spagnoli G., Grande A. M. and Di Landro L. Self-Healing Behavior of Blends Based on Ionomers with Ethylene/Vinyl Alcohol Copolymer or Epoxidized Natural Rubber. Macromolecular Materials and Engineering, 296, pp. 1119-1127 (2011).

[11] Rahman M. A., Penco M., Peroni I., Ramorino G., Grande A. M. and Di Landro L. Self-Repairing Systems Based on Ionomers and Epoxidized Natural Rubber Blends. ACS Applied Materials \& Interfaces, 3, pp. 48654874 (2011).

[12] Grande A. M, Rahman M. A, Di Landro L., Penco M., Spagnoli G. Self healing of blends based on Sodium Salt of Poly(Ethylene-co-Methacrylic Acid)/poly(ethylene-co-vinyl alcohol) and epoxidized natural rubber following high energy impact in Proceeding of 3rd International Conference on Self-Healing Materials. Bath, UK (2011).

[13] Grande A. M, Rahman M. A, Di Landro L., Penco M., Peroni I. Selfhealing response in different ionomer binary blends during projectile puncture tests in Proceeding of 4th European Conference for Aerospace Sciences, St. Petersburg, Russia (2011).

[14] A. Rahman, G. Spagnoli, A.M. Grande, L. Di Landro, "Role of Phase Morphology on the Damage Initiated Self-healing Behavior of Ionomer Blends", in Macromolecular Materials Engineering, (2013) DOI10.1002/mame.201200399. In press.

[15] Sihn S. and Rice B. P. Sandwich construction with carbon foam core materials, Journal of Composite Materials, 37, pp. 1319-1336 (2003).

[16] Spradling D. and Guth R. Carbon foams. Advanced Materials \& Processes, 161, pp. 29-31 (2003).

[17] Janszen G. and Nettuno P. G. Implementation and Validation of a Strain Rate Dependent Model for Carbon Foam in Proceeding of 14th International Conference on Computational Methods and Experimental Measurements, Algarve, Portugal (2009).

[18] Janszen G. and Nettuno P. G. Impact Response and Ballistic Performances of Graphitic Foams in Proceeding of 3rd International Conference on Safety and Security Engineering (SAFE/09), Rome, Italy (2009).

[19] A.M. Grande, L. Castelnovo, L. Di Landro, C. Giacomuzzo, A. Francesconi, M. A. Rahman. Rate-dependent self-healing behavior of an ethylene-co-methacrylic acid ionomer under high-energy impact conditions. Journal of Applied Polymer Science. doi: 10.1002/app.39384, (2013). 
[20] A. Francesconi, C. Giacomuzzo, A.M. Grande, T. Mudric, M. Zaccariotto, E. Etemadi, L. Di Landro, U. Galvanetto. Comparison of self-healing ionomer to aluminium-alloy bumpers for protecting spacecraft equipment from space debris impacts. Advances in Space Research, 51(5), pp. 930940, (2012). 